On the onset of void swelling in pure tungsten under neutron irradiation : An object kinetic Monte Carlo approach

\title{
Castin, N.
}

2017-09

Castin , N , Bakaev , A, Bonny , G , Sand , A E , Malerba, L \& Terentyev, D 2017 , ' On the onset of void swelling in pure tungsten under neutron irradiation : An object kinetic Monte

Carlo approach ' , Journal of Nuclear Materials , vol. 493 , pp. 280-293 . https://doi.org/10.1016/j.jnucmat.2017.06.00

http://hdl.handle.net/10138/307811

https://doi.org/10.1016/j.jnucmat.2017.06.008

cc_by_nc_nd

acceptedVersion

Downloaded from Helda, University of Helsinki institutional repository.

This is an electronic reprint of the original article.

This reprint may differ from the original in pagination and typographic detail.

Please cite the original version. 


\title{
On the onset of void swelling in pure tungsten under neutron irradiation: an object kinetic Monte Carlo approach
}

\author{
N. Castin ${ }^{\mathrm{a}}$, A. Bakaev ${ }^{\mathrm{a}}$, G. Bonny ${ }^{\mathrm{a}}$, A. E. Sand ${ }^{\mathrm{b}}$, L. Malerba ${ }^{\mathrm{a}}$, D. Terentyev ${ }^{\mathrm{a}}$ \\ ${ }^{a}$ Studie Centrum voor Kerneenergie - Centre d'Études de l'énergie Nucléaire (SCK•CEN), NMS unit, Boeretang 200, B2400, Mol, Belgium. \\ ${ }^{b}$ Department of Physics, University of Helsinki - P.O. Box 43, FI-00014 Helsinki, Finland.
}

\begin{abstract}
We propose an object kinetic Monte Carlo (OKMC) model for describing the microstructural evolution in pure tungsten under neutron irradiation. We here focus on low doses (under $1 \mathrm{dpa}$ ) where transmutation can be neglected. The emphasis is mainly centred on an adequate description of neutron irradiation, the subsequent introduction of primary defects, and their thermal diffusion properties. Besides grain boundaries and the dislocation network, our model includes the contribution of carbon impurities, which are shown to have a strong influence on the onset of void swelling. Our parametric study analyses the quality of our model in detail, and confronts its predictions with experimental microstructural observations with satisfactory agreement. We highlight the importance for an accurate determination of the dissolved carbon content in the tungsten matrix, and we advocate for accurate description of atomic collision cascades, in light of the sensitivity of our results with respect to correlated recombination.
\end{abstract}

Keywords:

Tungsten, Kinetic Monte Carlo, Neutron Irradiation, Void Swelling

\section{Introduction}

Tungsten is currently the officially selected constituent material for the divertor in ITER, and also for the first wall armour in the DEMO project $[1,2,3]$. This choice is mainly guided by its high melting point, high strength and high resistance to sputtering. In these reactors, the plasma-facing material is foreseen to experience extreme conditions, including a high heat load (up to $20 \mathrm{MW} \mathrm{m}^{-2}$ in normal conditions), a high flux of bombarding plasma particles (over $10^{24} \mathrm{~m}^{-2} \mathrm{~s}^{-1}$ ) and, also, a high flux of fast neutrons emitted from the fusion reactions [4]. In spite of the above-mentioned advantageous interaction properties with plasma, however, the intrinsic brittleness of tungsten can be regarded as the major safety issue, in particular for concepts including water-cooled divertors [5]. Even non-irradiated tungsten, for instance, is characterized with a ductile-to-brittle transition temperature (DBTT) as high as $300-400^{\circ} \mathrm{C}$ [6]. While this transition appears to be originally determined by the thermal activation of screw dislocations, neutron irradiation continuously generates lattice defects that further obstruct their glide, thus shifting the DBTT to even higher values. In any case, operation below DBTT induces a risk of cracking for plasma-facing components, because they cannot dissipate thermal stresses by plastic deformation [7]. If plasma damage is associated to a rather narrow penetration depth (around a few tens of micrometers as revealed by direct TEM investigations $[8,9]$ ), neutron irradiation, in contrast, affects the whole components resulting in a nearly homogeneous damage rate; the consequent microstructure pattern should, nevertheless, be strongly heterogeneous be-

Email address: nicolas.m.b.castin@gmail.com (N. Castin) cause of the temperature gradient from the plasma-facing surface towards heat sinks [10]. Undoubtedly, the present lack of consistent data on the performance of tungsten with respect to heat loads and transients under neutron irradiation remains to be filled in.

Accurate knowledge and understanding of the microstructural evolution of tungsten under neutron irradiation is an essential pre-requisite to rationalize and predict the resulting changes in its mechanical properties. For these reasons, such investigations are nowadays a "hot topic" in the field of fusion material development $[11,12]$. In substitute to full-scale fusion material testing facilities, experimental studies are conducted with fission test reactors $[13,14,15,16,17]$ in spite of the intrinsic differences in the neutron spectra involved. To start with, the thermal-to-fast neutrons ratio is, naturally, much higher in fission reactors, with consequences on the rate of transmutation to rhenium or osmium. Secondly, because of the presence of $14 \mathrm{meV}$ neutrons, the transfers of kinetic energy from impinging neutrons to primary knock-on atoms, with subsequent occurrence of atomic collision cascades, largely differ between fission and fusion reactors. This has a significant impact on the primary production of damage, and thus also potentially on the long-term evolution of the microstructure, as discussed later in section 2.3. Undoubtedly, these discrepancies in experimental set-ups, and therefore the underlying questions of transferability of experimental evidences from fission to fusion environments, imply a need for complementary computer-based modelling activities, where the design-relevant informations about the neutron spectrum and transmutation rates are adequately embedded.

Experimental evidence $[16,17,18]$ highlights that the mi- 
crostructure of neutron-irradiated tungsten (for the dose $0.1-1$ dpa) is primarily populated by dislocation loops and voids. Later, from $0.5 \mathrm{dpa}$ and above, non-coherent rhenium precipitates are generally observed. It thus appears from these observations that transmutation to rhenium and osmium due to thermal neutrons, and its potential subsequent effects, may only become significant from moderate doses, i.e., when the primary microstructure in terms of loops and voids is already established. Focussing on this latter aspect alone can thus be seen as an adequate first step towards the general objective of proposing a full model for neutron irradiation in tungsten. For these reasons, this work mainly focusses on the first stages of irradiation, i.e., when transmutation, and also the production of $\mathrm{H}$ or He, can be neglected as a reasonable approximation. Given the technological purity, however, the model must account for the presence of interstitial impurities (such as carbon), as well as for the polycrystalline nature of the material. Besides these aspects, the most important features to incorporate in the model are thus the atomic collision cascades, and the subsequent annealing and growth of the associated lattice defects.

The model we propose in this paper employs a regular object kinetic Monte Carlo (OKMC) method, as described in section 2. It takes steps from earlier works originally developed to describe neutron irradiation in BCC iron and iron-based alloys $[19,20]$. The parametrisation is adopted in accordance with the available knowledge based on previous studies, in particular, as implemented by C. Becquart and C. Domain in their "LAKIMOKA" code [21]. Here, we used the in-house code "MATEO", taking the same fundamental hypotheses in terms of lattice-defect description, occurrence of irradiation, elements of microstructure, etc. As explained later in section 2.5, the absence of solute atoms essentially simplifies the description of self-interstitial atoms (SIA) and their clusters; Considering their fast 1-D migration and given that relatively low irradiation doses are considered, the mutual long-range elastic interactions between SIA clusters may be disregarded. Also, simplifying assumptions can be taken at key moments of the simulation, in order to enhance the evolution of the physical time in the simulation box without fundamental alterations of the KMC algorithm. Next, considering that the primary attention is given to the description of vacancy-type defects, we propose a new parametrization by conducting complementary atomistic kinetic Monte Carlo (AKMC) simulations to carefully assess their thermal stability and diffusivity. Finally, the results of our model are presented in section 3, and discussed in the light of experimental data proposed by Tanno et al. in Ref. [14, 15]; Technically pure tungsten was irradiated in the Japanese JOYO reactor, which delivers a neutron spectrum close to the one expected in fusion environments (and therefore limited transmutation to rhenium or osmium takes place). The formation of voids is reported in a large range of temperatures $\left(400^{\circ} \mathrm{C}, 538^{\circ} \mathrm{C}\right.$ and $740^{\circ} \mathrm{C}$ ) and accumulated doses from $\sim 0.2$ up to $\sim 1.6 \mathrm{dpa}$. Undoubtedly, these experiments represent a rich set of data for the validation of our model. Further on, we explore the transferability of our model to higher irradiation temperatures, referring to another set of experimental data from Rau et al. [22] after neutron irradiation at $1000^{\circ} \mathrm{C}$. Globally, we perform a de- tailed sensitivity study to assess the impact of several key input parameters, such as: grain size, carbon content, and the spectrum of the kinetic energy transferred to the primary knock-on atom.

\section{Object kinetic Monte Carlo model}

As already mentioned, our object kinetic Monte Carlo (OKMC) model is originally inspired by an existing one proposed earlier by Bequart et al., as implemented in their LAKIMOKA software. Our new home software, called "MATEO", takes the same fundamental hypotheses as already extensively described in Ref. [21] and references therein. The model is however adapted, and some modifications are brought to comply for the specificities of the target experiments in this work. In the remaining of this section, we summarize the most important aspects of the model in view to prepare the discussions later in section 3, and, also, stressing the novelties.

\subsection{Simulation box, grain boundaries and dislocations}

Our simulation box embodies a pure tungsten matrix, with an eventual small content of chemical impurities assumed to be exclusively carbon, as explained in section 2.2. It is, at the beginning of the simulation, initially empty of irradiation defects (vacancies and self-interstitials), whereas regular microstructural features such as dislocations and grain boundaries are indirectly included, as described in what follows. Besides these, the box is shaped in a rectangular manner, and periodic boundary conditions (PBC) are applied in all directions to mimic an infinite, bulk bcc material. The edges are aligned with the $\langle 100\rangle$ directions, letting thus the $\langle 111\rangle$ directions be non-perpendicular to the PBC planes. Regarding the presence of one dimensionally (1-D) migrating defects along these $\langle 111\rangle$ directions (SIA clusters as explained later in section 2.4), an adequate aspect ratio for the box dimensions must be chosen in order to guarantee a realistic description of their probability of interaction with the other defects (vacancy clusters and/or voids), described as spherical absorbers in Ref. [23]. Cubicshaped boxes, for instance, or also rectangular-shaped with integer aspect ratios, implement and artefactual situation where $\langle 111\rangle$ directions are aligned with themselves while crossing the $\mathrm{PBC}$, thereby restraining the effective travelling in the simulation volume. We conducted relevant theoretical studies (not reported here for the sake of conciseness) to verify that an aspect ratio of 1.5 adequately counteracts this negative effect. Concretely, considering on the one hand that too big a simulation box is not manageable from the computing time point of view, but on the other hand regarding the order of magnitude for the defects densities in the target experiments in this work, we opted for the following box size: $120 a_{0} \times 180 a_{0} \times 270 a_{0}$, i.e., $38.0 \mathrm{~nm} \times 57.1 \mathrm{~nm} \times 85.6 \mathrm{~nm}\left(a_{0}=3.17 \AA\right)$, containing 11.664 million lattice sites. The minimal defect density that can be described, i.e., when no more than one defect of a given kind is found in the box, is thus $5.38 \cdot 10^{21} \mathrm{~m}^{-3}$.

Nevertheless, our simulation box is about three orders of magnitudes smaller than the grain sizes found in real materials (ranging from 5 to $50 \mu \mathrm{m}$ ). Grain boundaries may however 
be accounted for in an indirect and approximative way. In brief, it is assumed that grain boundaries act as theoretically perfect sinking elements for any kind of defects reaching it, and more complex reactions such as trapping or emission are not considered. This can be regarded as a reasonable assumption with little impact on the obtained results. After every migration event of a point-defect cluster (containing $n_{V}$ vacancies or $n_{I}$ SIA), a probability to have reached the grain boundary is calculated on the basis of the associated sink strength, as described in Ref. [23].

$$
\begin{aligned}
& k_{G B, 1 D}^{2}=\frac{15}{R_{G B}^{2}} \\
& k_{G B, 3 D}^{2}=\frac{14.4}{R_{G B}^{2}}
\end{aligned}
$$

Here, $k_{G B, 1 D}^{2}$ is the effective sink strength for 1-D migrating defects, whereas $k_{G B, 3 D}^{2}$ applies to 3-D migrating defects, and $R_{G B}$ is the grain radius (i.e., half of the so-called "grain size"). These expressions are based on the assumption that grains are spherically shaped, and that our simulation box represents an average population of defects being homogeneously distributed in it. It is worth noting that both formulas are almost identical from a numerical point of view, and also that the radius of interaction of the migrating defect is not explicitly written: it is in fact neglected compared to $R_{G B}$. Theoretically speaking, the above equations provide an estimate for the square of the inverse of the average distance travelled by the migrating defect before interaction, which can be related to the average number of jumps using:

$$
k^{2}=\frac{2 n}{d_{j}^{2}\left\langle n_{j}\right\rangle}
$$

Here, $n=1$ or 3 is the dimensionality of the jump, $d_{j}=$ $\sqrt{(3)} / 2 a_{0}$ is the jump distance, i.e., the first-nearest-neighbour distance, and $\left\langle n_{j}\right\rangle$ is the average number of jumps before interaction. In turn, the interaction probability after migration is given by:

$$
p_{G B}=\frac{1}{\left\langle n_{j}\right\rangle}=\frac{k_{G B}^{2} d_{j}^{2}}{2 n}
$$

Similarly, accurate description of the dislocation network cannot practically be implemented in our simulation box: including just one line sink playing the role of a single dislocation would, unavoidably, surpass by about two orders of magnitudes the dislocation density expected in real materials. Similarly to the case of the grain boundary, we make the assumption that dislocations are perfect sinks for any kind of defects, and a sinking probability $p_{d}$ after every migration event is calculated using Eq. 4 on the basis of the theoretical sink strength $k_{d}^{2}$ taken from Ref. [24]:

$$
\begin{aligned}
k_{d, 1 D}^{2} & =2\left(\pi\left(r_{d}+r\left(n_{V}, n_{I}\right)\right) \rho_{d}\right)^{2} \\
k_{d, 3 D}^{2} & =\frac{2 \pi \rho_{d}\left(1-\rho^{2}\right)}{\ln (1 / \rho)-0.75+0.25 \rho^{2}\left(4-\rho^{2}\right)} \\
\rho & =\left(r_{d}+r\left(n_{V}, n_{I}\right)\right) \sqrt{\pi \rho_{d}} \\
p_{d} & =\frac{k_{d}^{2} d_{j}^{2}}{2 n}
\end{aligned}
$$

Here, $r_{d}$ is the dislocation core radius, $r\left(n_{V}, n_{I}\right)$ is the action radius associated to the migrating defect (a function of $n_{V}$ or $n_{I}$ as defined later in section 2.4), and $\rho_{d}$ is the dislocation density.

In our reference experimental data $[14,15]$, the tungsten hot rolled sheet provided by Plansee Ltd. was annealed at $1400^{\circ} \mathrm{C}$ in vacuum during 1 hour, in order to release gases and residual stresses. This treatment should result in a substantial reduction of the dislocation density, and improve the uniformity of grains. According to available studies [8], the dislocation density is expected to be $\rho_{d} \approx 10^{12} \mathrm{~m}^{-2}$ (typical core radii should be $r_{d}=2.25 a_{0}$ ), while the grain size should be within a range of $5-50 \mu \mathrm{m}$. Considering the above equations, one can appreciate that 1-D migrating SIA defects, if not recombined with vacancy defects in the simulation box, are very likely to be sunk at the grain boundary preferably to the dislocation network. Assuming, for argument sake, $r=a_{0}$, we get $p_{d} \approx 8 \cdot 10^{-13}$, whereas we get $p_{G B} \approx 9 \cdot 10^{-8}$ for $R_{G B}=5 \mu \mathrm{m}$ and $p_{G B} \approx 9 \cdot 10^{-10}$ for $R_{G B}=50 \mu m$. The radius $R_{G B}$ for the grain boundary is potentially a sensitive input parameter for the model, and its impact on our predictions of void swelling is thus investigated later in section 3.

\subsection{Chemical impurities}

In spite of high quality standards in the industry, and the relatively tiny content when given in units of weight-per-million (wppm), chemical impurities such as carbon cannot be overlooked from our modelling purposes, because of the significant role they might potentially play on the evolution of the microstructure during irradiation. It is indeed known from DFT calculations that carbon strongly binds with single vacancies and their clusters [21], and also with SIA's and their clusters $[21,25]$. Concretely, this translates into the OKMC model as a dual effect: (a) By adding a surplus activation energy to overcome the binding, carbon atoms temporarily prevent the migration of defects being in interaction with them, and thus significantly alter the overall kinetics of thermally activated diffusion, with possible consequences in the rate of seeding, coarsening and growth of vacancy clusters; (b) In addition, they inhibit the release of single vacancies from the formed clusters, thereby enhancing their stability and thus, also, affecting the process of voids formation.

A starting point for the adequate inclusion of these reactions in our OKMC model is the determination of the content of carbon being dissolved in the tungsten matrix, before neutron irradiation begins. The global content in samples provided by Plansee Ltd., for instance, were reported in Ref. [15] in the 40-200 wppm range, but more accurate estimations based on chemical analysis [26] concluded for $17 \mathrm{wppm}$. In term of atomic proportions, this latter concentration corresponds to 260 atomic-per-million (appm). It means, in practical terms, that a total number of 3032 carbon atoms should, somehow, be included in the volume corresponding to our simulation box. These atoms are not necessarily dissolved in the matrix, however, because of the strong affinity of carbon to dislocations and grain boundaries. According to available DFT data, the binding energy of carbon to a high angle grain boundary is as large as 3 $\mathrm{eV}$ [27]; a substantial proportion of the overall carbon content 
might thus potentially be found in grain boundaries, and does thus virtually not interfere with the migration and dissolution of vacancy defects in the bulk. To our best knowledge, there is no direct experimental evidence that can be exploited to estimate the proportion of carbon that remains dissolved in the matrix. We thus consider a few cases, assuming that this concentration varies from 0 (thus being the carbon-free limiting case) to 50 appm.

If included in the simulation box, accurate modelling of the interactions between interstitial carbon atoms and vacancies or SIA defects is rather delicate to implement in practice, for at least two main reasons. First of all, interstitial carbon atoms are relatively mobile elements, since the associated migration energy is comparable to the one associated to single vacancies. Including them explicitly in the model, under the form of individual defects each assigned with their own migration events, would unavoidably and dramatically alter the advancement of the physical time during the execution of the KMC simulation. Following the mean residence time principle $[28,29,30]$, the average time increment $\Delta t_{M C}$ after any event is inversely proportional to the sum of all events frequencies: $\Delta t_{M C} \sim 1 / \Gamma_{t o t}=1 /\left(\sum_{e} \Gamma_{e}\right)$, with $e$ being the event indices. The permanent presence of hundreds or thousands of carbon atoms would thus contribute as a constant, and immutable term in $\Gamma_{t o t}$, significantly decreasing $\Delta t_{M C}$. Secondly, and most importantly, the number of possible reactions in the model would become unmanageable, especially regarding the large number of input parameters necessary to parametrize them. For these reasons, past works [31] have taken a simplifying assumption to substitute explicit carbon atoms for an equivalent set of fixed trapping elements, easier to manage from both the algorithmic and the computing points of view. Conceptually, these trapping elements can be regarded as a population of carbon $(\mathrm{C})$ and vacancy (V) compounds, e.g., single $\mathrm{C}$ atoms, $\mathrm{CV}$ clusters, $\mathrm{C}_{2} \mathrm{~V}$ clusters, etc., that are presumably representative of the steady state found in the studied material, as a function of carbon content, temperature, and the flux of incoming vacancies. Ad-hoc and dedicated simulations, as e.g., described in Ref. [31], may be conducted to evaluate this steady state population, at the expense of a number of assumptions and simplifications. Next, the required input parameters to the OKMC model is a list of binding energies $E_{b}\left(n_{I}, n_{V}, t\right)$ between the identified trapping element $(t)$, and any point-defect cluster (containing $n_{I}$ SIA or $n_{V}$ vacancies):

$$
\begin{aligned}
\Gamma_{M i g}^{(t)} & =\Gamma_{M i g} \cdot \exp \left(\frac{-E_{b}\left(n_{I}, n_{V}, t\right)}{k_{B} T}\right) \\
\Gamma_{E m m}^{(t)} & =\Gamma_{E m m} \cdot \exp \left(\frac{-E_{b}\left(n_{I}, n_{V}, t\right)}{k_{B} T}\right)
\end{aligned}
$$

Here, $\Gamma_{M i g}^{(t)}$ is the reduced migration frequency associated to the point-defects cluster when interacting with trap $t$, whereas $\Gamma_{M i g}$ is the value otherwise (in tungsten matrix). Similarly, $\Gamma_{E m m}^{(t)}$ is the reduced frequency for the emission of a single point-defect from the cluster.

The end-results of the above-described study is, nevertheless, an approximate yet finely tuned scheme, whose elabo- ration makes full sense only for being implemented in welladvanced models, already intensively optimized for the studied materials. This work is, from our perspective, a first step towards the definition of an OKMC model for neutron irradiation in tungsten, and many required input parameters for describing $\mathrm{C}-\mathrm{V}$ interactions have not been carefully evaluated with DFT yet. At this preliminary stage, we thus opted for relying on even stronger assumptions, for the sake of simplicity. While a more accurate determination for an adequate population of trapping elements, and the corresponding values for $E_{b}\left(n_{I}, n_{V}, t\right)$ are in principle required for future steps, we here included Cbased trapping elements in the following way: (a) A single kind of trap was defined, assumed to be isolated and single carbon atoms; (b) Since the concentration of carbon in the matrix is not accurately known, as discussed above, we analyse later in section 3 how the predictions of our OKMC model change when it varies from 0 to 50 appm; (c) Finally, we assumed that the binding energy $E_{b}\left(n_{I}, n_{V}, t\right)$ is $1 \mathrm{eV}$ for all SIA clusters $\left(n_{I}>0\right.$ and $n_{V}=0$ ) independently of their size. This value slightly overestimates DFT findings for binding energies between single carbon atoms and single SIA's (0.62 eV in Ref. [21] and 0.82 $\mathrm{eV}$ in Ref. [25]). More assumptions were made concerning vacancy clusters, however. We assumed value a binding energy of $1.50 \mathrm{eV}$ for the single vacancy defect $\left(n_{V}=1\right.$ and $\left.n_{I}=0\right)$, which is slightly lower than DFT values (around $2 \mathrm{eV}$ in Ref. $[21,25])$. Next, we assumed that the magnitude of binding decreases with the addiction of more vacancies in the cluster. This translates the assumption that if carbon atoms affect the binding of vacancies in their immediate vicinities, they cannot act with significant magnitude on the whole interface between the tungsten matrix and the vacancies composing a cluster. Concretely, and for the sake of simplicity, we assumed a linear decrease with the number of vacancies, postulating that if $n_{V} \geq n_{V}^{\left(B_{0}\right)}$, carbon atoms have no longer any significant effect on the migration or emission events. The effective binding energy between C-based trapped and point-defect clusters as assumed in our OKMC model thus writes, in units of $\mathrm{eV}$ :

$$
\begin{aligned}
& E_{b}\left(n_{I}>0, n_{V}=0, t\right)=1 \\
& E_{b}\left(n_{I}=0, n_{V}>0, t\right)=1.5\left(1-\frac{n_{V}-1}{n_{V}^{\left(B_{0}\right)}-1}\right)
\end{aligned}
$$

Similarly to grain boundaries and dislocation networks as described in section 2.1, we opted for a stochastic description of the trapping elements instead of the explicit introduction of fixed and spherical objects in the simulation box. Given the concentration and the capture radius $R_{t}$ associated to the traps, the probability $p_{t}$ of interaction with point-defect clusters (with radius $r\left(n_{V}, n_{I}\right)$, as defined later in section 2.4), after a migration event, is calculated on the base of the corresponding sink strength $k_{t}$, as provided in Ref. [23]:

$$
\begin{aligned}
k_{t, 3 D}^{2} & =4 \pi N_{t}\left(r_{t}+r\left(n_{V}, n_{I}\right)\right) \\
k_{t, 1 D}^{2} & =2\left(\pi\left(r_{t}+r\left(n_{V}, n_{I}\right)\right)^{2} N_{t}\right)^{2} \\
p_{t} & =\frac{k_{t}^{2} d_{j}^{2}}{2 n}
\end{aligned}
$$


Here, $k_{t, 1 D}^{2}$ is the sink strength for $1 \mathrm{D}$ migrating defects, $k_{t, 3 D}^{2}$ is for $3 \mathrm{D}$ migrating ones, and $N_{t}$ is the trap density. Consistently with past work, and without loss of generality, we assumed $R_{t}=1.75 a_{0}$. To fix ideas, assuming 1-D migration and, again for argument sake, that $r=a_{0}$, the above expressions lead to the following probabilities: $p_{t}=2 \cdot 10^{-7}$ if $N_{t}=10$ appm and $p_{t}=4 \cdot 10^{-6}$ if $N_{t}=50 \mathrm{appm}$. Needless to say, these probabilities are much larger than these associated to the grain boundary sinking event, as derived in the previous section; the presence of carbon in the simulation box, even in small concentration, is thus expected to substantially delay the onset of voids, because the probability for SIA to reach the grain boundary is largely reduced, and so its chances to get recombined with vacancies increase.

\subsection{Neutron irradiation}

Neutron irradiation is described as a set of stochastic events, each assigned with a frequency of occurrence adequately calculated in order to implement a desired dose rate (in dpa/s), as explained in what follows. When selected and applied, a whole group of point-defect clusters is included in the simulation box with no time increment, playing the role of debris remaining after the occurrence of an atomic collision cascade. In the real material, the latter is triggered by a neutron hitting a primary knock-on atom (PKA) with a high enough transfer of kinetic energy (about $90 \mathrm{eV}$ for tungsten) to generate stable damage, i.e., at least a Frenkel pair that doesn't spontaneously annihilate without the need for thermally activated migration.

Because it is virtually inconceivable to observe the occurrence of such an event with an experimental technique, there are practically no other ways to study accurately cascade debris than performing computer simulations at the atomic scale, e.g., using molecular dynamics methods based on interatomic potentials as thoroughly presented in Ref. [32, 33]. Given as main input parameter the kinetic energy transferred from the impinging neutron to the primarily collided atom, henceforth referred to as "PKA energy" (PKAE) and denoted as $E^{(P K A)}$, several independent simulations are conducted, and the remaining population of debris is investigated: any imperfection in the otherwise undisturbed bcc structure is catalogued, in general terms, as a point-defect or a point-defect cluster, described by spatial coordinates (relative to the PKA) and a composition $\left(n_{V} \geq 1\right.$ or $\left.n_{I} \geq 1\right)$. For single SIA or SIA clusters $\left(n_{I} \geq 1\right)$, however, additional information regarding the orientation was also recorded; using a maximum likelihood principle, they were labelled with one of the four $\langle 111\rangle$ directions in the bcc crystallographic structure, thus providing accurate information about the relative orientation with respect to the neighbouring defects. Naturally, the higher the PKAE, the more and the bigger stable debris are found, as illustrated in Fig. 1. Individual Frenkel pairs are the usual debris after low PKAE cascades $(\leq 5 \mathrm{keV})$, whereas clusters of both vacancies or SIA are clearly formed when higher energies are involved. Specifically in the case of tungsten, because of the rarer occurrence of branching during the cascade compared to the case of iron, large vacancy clusters identifiable as small voids ( $\geq 50$ vacancies) may be formed
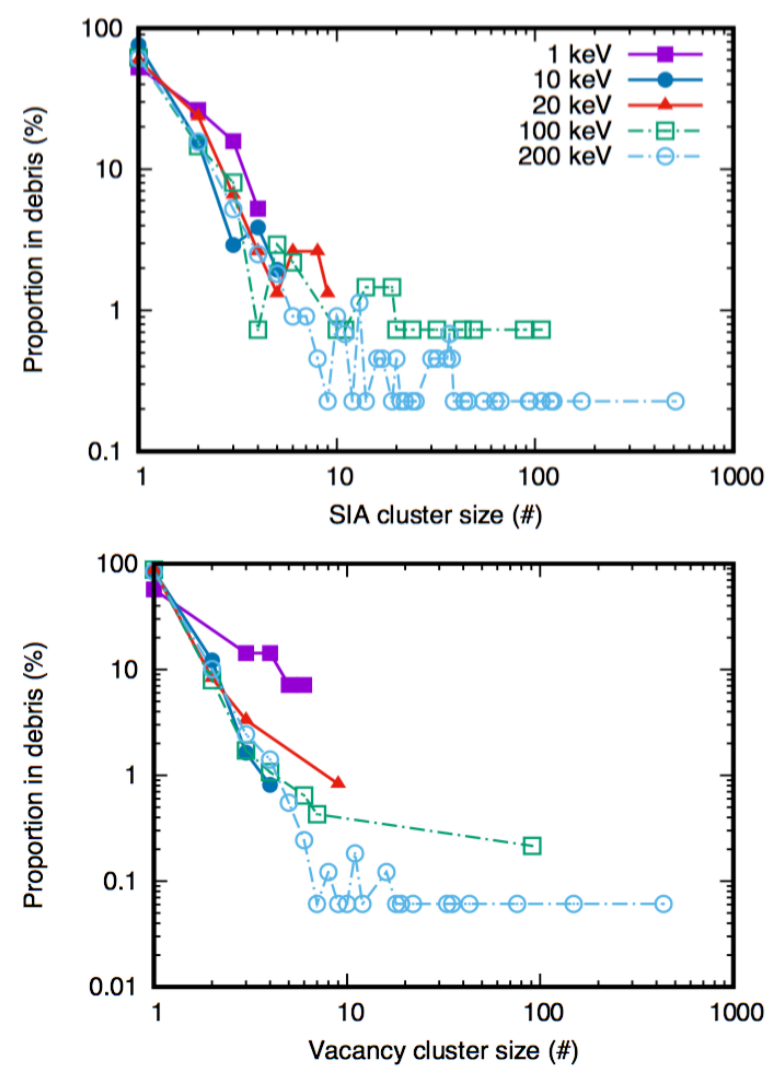

Figure 1: Proportion of sizes for point-defect clusters found in MD-based cascade debris, at various PKAE. The top figure shows SIA clusters, and the bottom one shows vacancy clusters. Series in plain line correspond to PKAE within the JOYO spectrum shown in Fig. 2, whereas the dashed ones are only included in the homogeneous spectrum.

from $E^{(P K A)} \approx 100 \mathrm{keV}$, and so do SIA clusters big enough to be considered as small dislocation loops ( $\geq 90$ SIA).

In past works [32, 33], a database of cascade debris was generated for a discrete set of PKAE values ranging from $90 \mathrm{eV}$ up to $200 \mathrm{keV}$. Here, separate and independent irradiation events are defined in the OKMC model for each of these PKAE, with a frequency denoted as $\Gamma_{i r r}^{(P K A E)}$. They can be seen as a discrete approximation for a real, continuous spectrum found in actual nuclear reactors. The red lines in Fig. 2 show the distribution in PKAE estimated [34, 35] for the Japanese JOYO reactor, used for performing the neutron irradiation of the tungsten samples reported in the work by Tanno et al. [14, 15], whereas the dot points highlight which MD-based catalogues of debris are available. For each PKAE, an equivalent value of displacement-per-atom (dpa), hereby denoted as $d_{a t}$, is calculated using the standard NRT formula:

$$
d_{a t}\left(E^{(P K A)}\right)=\frac{0.8 E^{(P K A)}}{2 E_{d} N_{a t}}
$$

Here, $E_{d}=90 \mathrm{eV}$ is the displacement threshold for tungsten, and $N_{a t}=11.664 \cdot 10^{6}$ is the total number of atoms in our simulation box. In practice, the global desired dose rate $D_{a t}$ is a given input for the OKMC simulation. It can be derived as:

$$
D_{a t}=\sum_{P K A E} \Gamma_{i r r}^{(P K A E)} d_{a t}\left(E^{(P K A)}\right)
$$




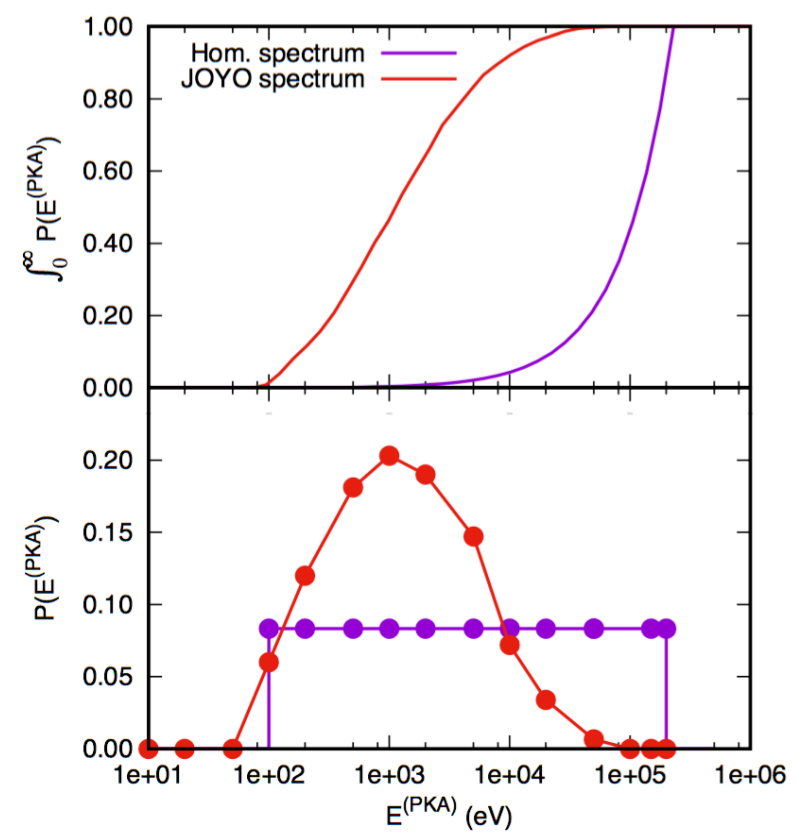

Figure 2: PKAE spectra for the JOYO reactor or in a fictive homogeneous condition. Dots show for what values debris libraries were obtained with MD. (top) Cumulative spectra; (bottom) Probability density functions for each PKAE.

Here, the summation is performed on all values of PKAE for which debris libraries are available. An effective average displacement-per-atom may be defined as:

$$
\left\langle d_{a t}\right\rangle=\sum_{P K A E} P\left(E^{(P K A)}\right) d_{a t}\left(E^{(P K A)}\right)
$$

Here, $P\left(E^{(P K A)}\right)$ is the probability for the occurrence of $E^{(P K A)}$ in the spectrum, as shown in the lower panel in Fig. 2. We next derive:

$$
\Gamma_{i r r}^{(P K A E)}=\frac{D_{a t} P\left(E^{(P K A)}\right)}{\left\langle d_{a t}\right\rangle}
$$

The introduction of irradiation-produced defects in our simulation box with the method described in this section is fully consistent with the goals and framework of the OKMC model. It aims at incorporating relevant information explicitly derived in atomic-scale models for an accurate description of the early stages of irradiation. For instance, no excessive assumption is made with respect to the number of defects introduced after each cascade, neither concerning their size, nor their relative position and orientation. It is worth emphasizing that these two latter points carry a significant importance in the accurate description of irradiation, because they have a strong influence on the rate of correlated SIA-vacancy recombination. Point-defects of opposite nature are indeed not created homogeneously in space, but should rather be found in a logical sequence that naturally emerges from a collision cascade: in simple and pictorial words, since atoms are pushed towards a given direction, vacancies are found in the beginning of a branch, whereas SIA are found at the end of it. This production bias unavoidably governs the probability for correlated recombination, i.e., the chances that SIA's encounter vacancies coming from the same cascade after few migration events, and disregarding it may potentially affect the overall predictions of the model. On the other hand, the major drawback of the approach is that the finite number of MD simulations performed may provide limited, potentially incomplete statistics, from at least two perspectives: (a) given the PKAE, one can appreciate substantial variations in the resulting population of debris. As a matter of fact, some MD runs lead to substantially less defects, while others create substantially more, and in particular much bigger individuals. Bearing in mind that point-defect clusters of increasing sizes do not have the same long-term impact on the overall microstructure (in other words, one big cluster is by no means equivalent to two half-sized ones), this can be regarded, in the limiting case, as a potential source of inaccuracy for the description of irradiation ; (b) In spite of the theoretical spectrum shown in Fig. 2, characterized by a hypothetical maximal value, a higher PKAE might in fact occasionally occur. Considering the previous discussion, it also means that big point-defect clusters might be included directly from the collision cascades, even though occasionally, and even if it is a priori not anticipated from the theoretical PKAE spectrum.

In light of the above discussions, an alternative approach to the finite library of cascade debris could thus be to rather rely on a "statistical introduction" of defects in the simulated volume. Given the PKAE for a given irradiation event, a corresponding number and size distribution of point-defect clusters may be randomly selected, based on an adequately established scheme fitted from MD simulations. In the absence of another valid option, these defects are then included in the simulation box at random positions. These can be eventually limited within a given volume near the PKA, or in the limiting case within the whole simulation box for a more consistent approach. In one or the other case, however, the immediate and unavoidable downside is that the production bias is either approximated, or totally neglected. To get insights from our model, we thus investigate later in section 3 the following aspects:

- The sensitivity of our model predictions with respect to correlated recombination. Our results are compared when neutron irradiation is described using the MD-obtained libraries of cascade debris, or the statistical defects assumption. To limit the number of varying parameters from one series of results to the other, the latter are implemented in the simplest way. To ensure that the number of defects and the size distribution are rigorously identical, the MDobtained libraries of cascade debris are taken as a source of defects, but every individual point-defect cluster is included in a randomly chosen position in the whole simulation box. This study thus focusses on the sole sensitivity of our model with respect to the accurate account of the production bias.

- The impact of the highest PKAE on the obtained results. We thus consider separately the two spectra depicted in Fig. 2. In addition to the one estimated for the JOYO 
reactor, we also consider the limiting case of a totally homogeneous spectrum, ranging from $E_{d}$ to the maximum of $200 \mathrm{keV}$ that was simulated with MD. Concretely, the main difference between both spectra is that the homogeneous one includes significantly bigger defects after each irradiation event, as can be appreciated in Fig. 1.

\subsection{Point-defect clusters}

Following the terminology used through this paper, "pointdefect clusters" denote any cluster of either vacancies or SIA's, including the single ones for the sake of generality. They are gradually introduced in our simulation box when neutron irradiation events are applied. We assume that they always have a spherical shape, independently of their size or nature; SIA loops, for example, are thus not explicitly cared of as such in our model. Similarly to past works[31], the radius $r\left(n_{V}, n_{I}\right)$ is defined in the following way, in units of lattice parameters $a_{o}$ :

$$
\begin{aligned}
& r\left(n_{V}>0, n_{I}=0\right)=\frac{D_{R e c}}{1+\gamma}+\varepsilon+\left(\frac{3}{8 \pi}\right)^{1 / 3}\left(n_{v}^{1 / 3}-1\right)(20) \\
& r\left(n_{V}=0, n_{I}>0\right)=\frac{\gamma D_{R e c}}{1+\gamma}+\frac{\sqrt{n_{I}}-1}{\sqrt{\pi \sqrt{3}}}
\end{aligned}
$$

Here, $\varepsilon=0.01$ is a small positive value, $\gamma=1.2$ is a biasing factor in favour of SIA, and $D_{R e c}$ is the so-called recombination distance, i.e., the maximal distance that may separate a single SIA from a single vacancy for the occurrence of mutual annihilation. Consistently with past works [21], we opted for a small value, corresponding to the $1 \mathrm{nn}$ distance: $D_{R e c}=a_{o} \sqrt{3} / 2+\varepsilon$. Possible events assigned to point-defect clusters are essentially identical, also. To start with, both kinds have a migration event. Vacancy clusters jump to one of their eight $1 \mathrm{nn}$ positions, randomly chosen with equal probability, whereas SIA clusters migrate either forwards or backwards along their present Burgers vector. In the case of a single SIA, a rotation event is systematically considered before the migration is applied with a probability estimated on the basis of a rotation energy of $0.38 \mathrm{eV}$. SIA clusters, however, are assumed to have no rotation event and are thus fully 1-D migrating objects. The migration frequency is estimated with a classical Arrhenius expression:

$$
\Gamma_{m i g}\left(n_{v}, n_{I}\right)=\Gamma_{m i g}^{(0)}\left(n_{v}, n_{I}\right) \exp \left(\frac{-E_{m i g}\left(n_{v}, n_{I}\right)}{k_{B} T}\right)
$$

Here, $\Gamma_{m i g}^{(0)}\left(n_{v}, n_{I}\right)$ is a size and composition dependent prefactor, whereas $E_{m i g}\left(n_{v}, n_{I}\right)$ is an equally dependent migration energy. Next, while SIA clusters are assumed in first approximation to be indissoluble, vacancy clusters, however, have a dissociation event, during which a single vacancy is emitted from the cluster and is placed in a nearby location. The frequency of occurrence writes:

$$
\Gamma_{\text {diss }}\left(n_{v}\right)=\Gamma_{\text {diss }}^{(0)}\left(n_{v}\right) \exp \left(\frac{-E_{\text {diss }}\left(n_{v}\right)}{k_{B} T}\right)
$$

Again, $\Gamma_{\text {diss }}^{(0)}\left(n_{v}\right)$ is a size-dependent prefactor, and $E_{\text {diss }}\left(n_{v}\right)$ is a size-dependent dissociation energy.
Our parametrization for SIA clusters was taken from Ref. [21] without changes; the prefactor $\Gamma_{\text {mig }}^{(0)}\left(n_{v}=0, n_{I}>0\right)$ decreases exponentially from Debye's frequency following a negative power-law of $n_{I}$, and the migration energy is constant: $E_{\text {mig }}\left(n_{v}=0, n_{I}>0\right)=0.013 \mathrm{eV}$. The parameters for vacancy clusters were, however, obtained with an original method in this work. Following the methodology presented in Ref. [36, 37], we conducted series of atomistic kinetic Monte Carlo (AKMC) simulations to study the process of migration and dissociation of vacancy clusters $\left(n_{v}=2, \ldots, 250\right)$, in a large temperature range (from $640 \mathrm{~K}$ to $2000 \mathrm{~K}$ ). In brief, an artificial neural network (ANN) was trained to predict how individual vacancy migration energies vary according to their local atomic environment, as calculated using the nudge elastic band [38] method and using an interatomic potential by Marinica et al. [39]. It was then used as engine during the AKMC simulations, to provide on-the-fly and accurate estimates of the vacancy migration energies, taking thus indirectly the effect of long range relaxation into account in spite of the rigid lattice description of the atomic system. Migration or dissociation of the vacancy clusters did thus occur spontaneously, from the sequence of MCselected events, and was thus not further approximated.

The fitted Arrhenius coefficients for vacancy clusters are shown in Fig. 3. In addition to the obtained values for the particular clusters that were considered, the figure also shows simple extrapolative laws that were fitted. Unsurprisingly, we see in the top figures that the bigger the vacancy cluster, the lower the attempt frequency for migration. The migration energy increases, but saturates at $2.77 \mathrm{eV}$ from $n_{V}=100$ and on. This means that the vacancy clusters start behaving like small voids, and the local curvature at the $\mathrm{W}$-vac interface already gets binding properties close to a free surface. The bottom figures show the dissociation frequency. We see that the associated dissociation energy increases with the number of vacancies, also saturating from $n_{V}=100$ for the same reasons as discussed before. The dissociation prefactor, however, is clearly increasing without saturation. This translates the fact that while the binding energy of a single vacancy to the cluster has converged, the total surface at the $\mathrm{W}$-vac interface increases, and so does, logically, the frequency at which the emission of a single vacancy is attempted. Concretely, the resulting frequencies for the migration and dissociation events at the working temperatures in this work are shown in Fig. 4, in comparison with the parametrisation employed by Becquart et al. in Ref. [21]. The Y-axis have been chosen in such a way that the minimum visible value, $10^{-7} s^{-1}$, corresponds to one occurrence within 1 dpa irradiation time in the condition of Tanno et al.; only those many orders of magnitudes higher are thus considered important. Clearly, our parametrisation suggests that above $740^{\circ} \mathrm{C}$ both migration and dissociation events remain likely even for very big clusters and voids, contrarily to the capillarity model employed by Becquart et al.

\subsection{Simulation scheme and approximations}

The conditions, parameters and hypotheses expounded in the previous section adequately fulfil our objective to simulate the 


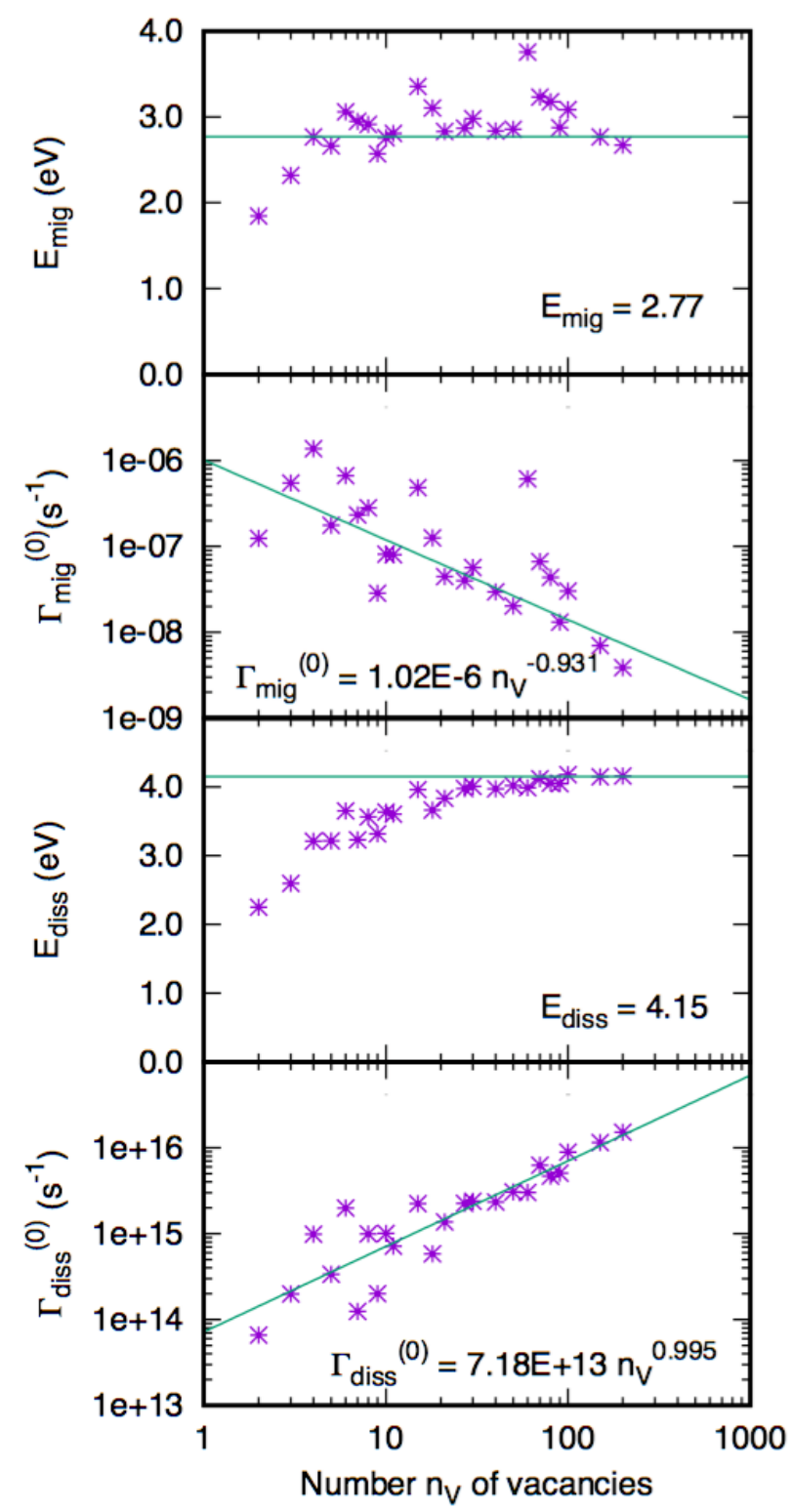

Figure 3: Parameters for vacancy clusters obtained with the ANN-based AKMC model. (top) Migration attempt frequency and the associated migration energy. (bottom) Prefactor for single vacancy emission and the associated dissociation energy. microctructural evolution in pure tungsten under neutron irradiation, focussing mainly on the onset of nanovoids formation during low doses. In the absence of minor alloying elements such as rhenium or osmium, with little or no carbon dissolved in the matrix, and, finally, neglecting the long-range elastic interactions between SIA's, the latter are destined to behave like "volatile" defects. Because their migration energy is of practically no significant magnitude, SIA clusters can, undoubtedly, be regarded as nearly zero-time migrating elements, and an OKMC simulation conducted in these conditions can be described in two succeeding stages:

1. When an irradiation event is selected and applied, thereby including new SIA clusters in the simulation volume, the list of MC events becomes unavoidably outbalanced between very fast, almost immediate events, and others whose likeliness to take place is negligible. In other words, almost exclusively migration events for SIA clusters are selected, until they either coalesce in very big clusters with reduced migration frequency, or vanish from the simulation box. This latter event can happen after either recombination with a vacancy cluster, absorption at grain boundary, or the far less likely absorption at the dislocation network.

2. Next, only vacancy clusters and, eventually, big SIA clusters remain in the simulation box. The following MC events thus let evolve the population of vacancy clusters, until the next occurrence of irradiation.

Clearly, the first above-mentioned stage largely dominates the OKMC simulation from the computing point of view. When included during an irradiation event, single SIA's rapidly annihilate (in term of number of MC events, via correlated recombination or not), or find another SIA cluster to merge, because of their 3-D motion. Regarding their 1-D motion, SIA clusters, however, are likely to perform a substantial amount of migration jumps (successions of forwards/backwards displacement along their Burgers vector) before they are found to interact with other defects. These circumstances largely inhibit the advancement of the simulation, especially for the largest grain boundary radii $R_{G B}$ that we considered, because a major proportion of MC events ( $\gg 99 \%$ ) that are selected and applied concern the migration of SIA clusters, at detriment of others that make the simulation time progress. The presence of trapping elements tends to bring a better balance in the MC events, because the simulation systematically passes by temporary states when all SIA clusters are caught by one of them, in which case the simulation time progresses thanks to the binding energies added to their migration energies.

Nevertheless, the sequential behaviour of our simulation can be exploited in order to alleviate the computing time, at the cost of slightly deviating from the strict application of the OKMC algorithm. When the status of the simulation is found to linger in the first stage, and when no more correlated recombination is expectable, we assume that it is reasonable to skip the intrinsic detailed sequence of events proper to the OKMC, and formally decide for a fate for all remaining fast-migrating SIA clusters immediately. Concretely, all of them are individually 


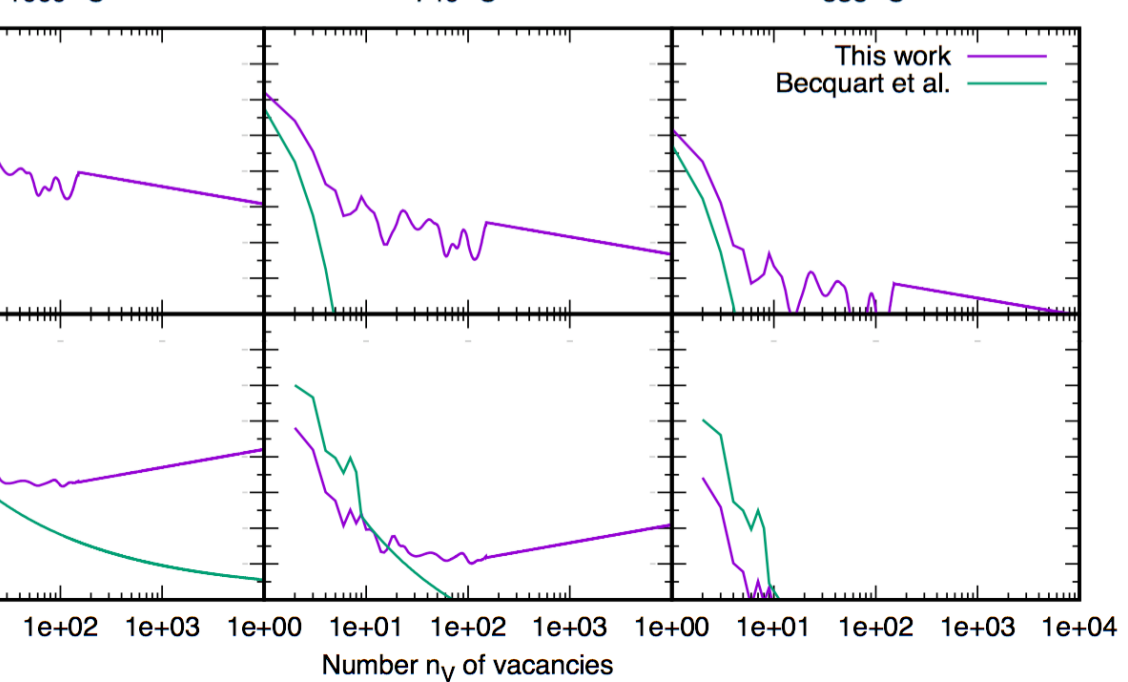

Figure 4: Frequencies for the migration and dissociation events assigned to vacancy clusters of increasing size, at three different temperatures. Our ANN-AKMC results are compared with the parametrisation based on capillarity laws by Becquart et al. in Ref. [21].

played in a MC selection of one in three events: Absorption by GB, absorption by dislocation, or recombination with one of the vacancy clusters in the box. Each probability is rigorously evaluated without approximation, using the theoretical expressions for the sink strength: Eq. 4 and 8 are directly applicable to the two first events, whereas recombination with a vacancy cluster is evaluated with an expression similar to Eq. 15. Strictly speaking, this approximated scheme can only be applied in carbon-free simulations, because the presence of trapping elements, even in small concentrations, would annihilate its benefits. Further on, we opted for a reasonable compromise between speed and accuracy, deciding that two more conditions must be met; (1) After an irradiation event has occurred, the number of successive events associated to SIA clusters must be larger than a given threshold (experience inspired us to chose for $10^{7}$ events); (2) The number of remaining fast-migrating SIA clusters must be small (not larger than 5).

\section{Results}

In this section, we analyse the predictions of our OKMC model and compare them with experimental evidences, in order to evaluate its overall ability to properly describe the onset of void swelling for low doses of neutron irradiation. In particular, several sets of input parameters are used to carefully assess the sensitivity of our results with respect to key parameters, as discussed through section 2. We start, in section 3.1, by exploring the carbon-free limiting case, i.e., assuming a chemically pure tungsten matrix. The basic trends and observations are thus drawn, providing a reference case for the assessment of the multiple input parameters of the model. Carbon-based trapping elements are then included in section 3.2, in order to appreciate the importance of accurate knowledge of the content of carbon diluted in the matrix. Finally, the parameters proper to neutron irradiation are explored in section 3.3 , in view to of the importance of an accurate description for correlated recombination. Globally, all performed simulations are centred around relevant conditions for the experiments by Tanno et al. [14, 15] and Rau et al. [22]. Our results are thus systematically evaluated at four different temperatures in a large range $\left(T=400^{\circ} \mathrm{C}, 538^{\circ} \mathrm{C}, 740^{\circ} \mathrm{C}\right.$ and $1000^{\circ} \mathrm{C}$ ). The quality of our model and its parametrization is thus adequately appreciated.

\subsection{Carbon-free tungsten limit}

Fig. 5 illustrates the results obtained with our model in terms of average void densities and average void sizes, in the carbonfree tungsten limit. Three different radii for the grain boundary were assumed: $R_{G B}=5,12.5$ or $25 \mu \mathrm{m}$. Experimental data points obtained by TEM from Tanno et al. [14, 15] and Rau et al. [22] are also included in the figure. The comparison is complemented in Fig. 6, where the distribution of void sizes are shown at various doses. At first glance, one can appreciate that our results are, globally, in fair agreement with the TEM observations, because both the density of observed voids and their average size seem to have followed the same trends with the irradiation temperature. Needless to say, a perfect match was not expectable. TEM observations are by no means directly comparable in a one-to-one fashion with our OKMC results, at least because of the intrinsic discrepancies in the analysis methods. Specifically, the population of smallest voids (around $1 \mathrm{~nm}$ size) is apparently a source of disagreement, as can be appreciated in Fig. 6. These voids are the most delicate to observe experimentally, whereas all of them may unequivocally been taken into account while analysing the content of our simulation box. It is worth emphasizing, however, that the problem does not pose itself for the highest irradiation temperature $\left(1000^{\circ} \mathrm{C}\right)$, because no such small voids seem to be stable. In light of this discussion, the results shown in Fig. 5 were processed ignoring voids smaller than $1.5 \mathrm{~nm}$ in size in order to make a more relevant comparison. Qualitatively, we see that the overall trend 

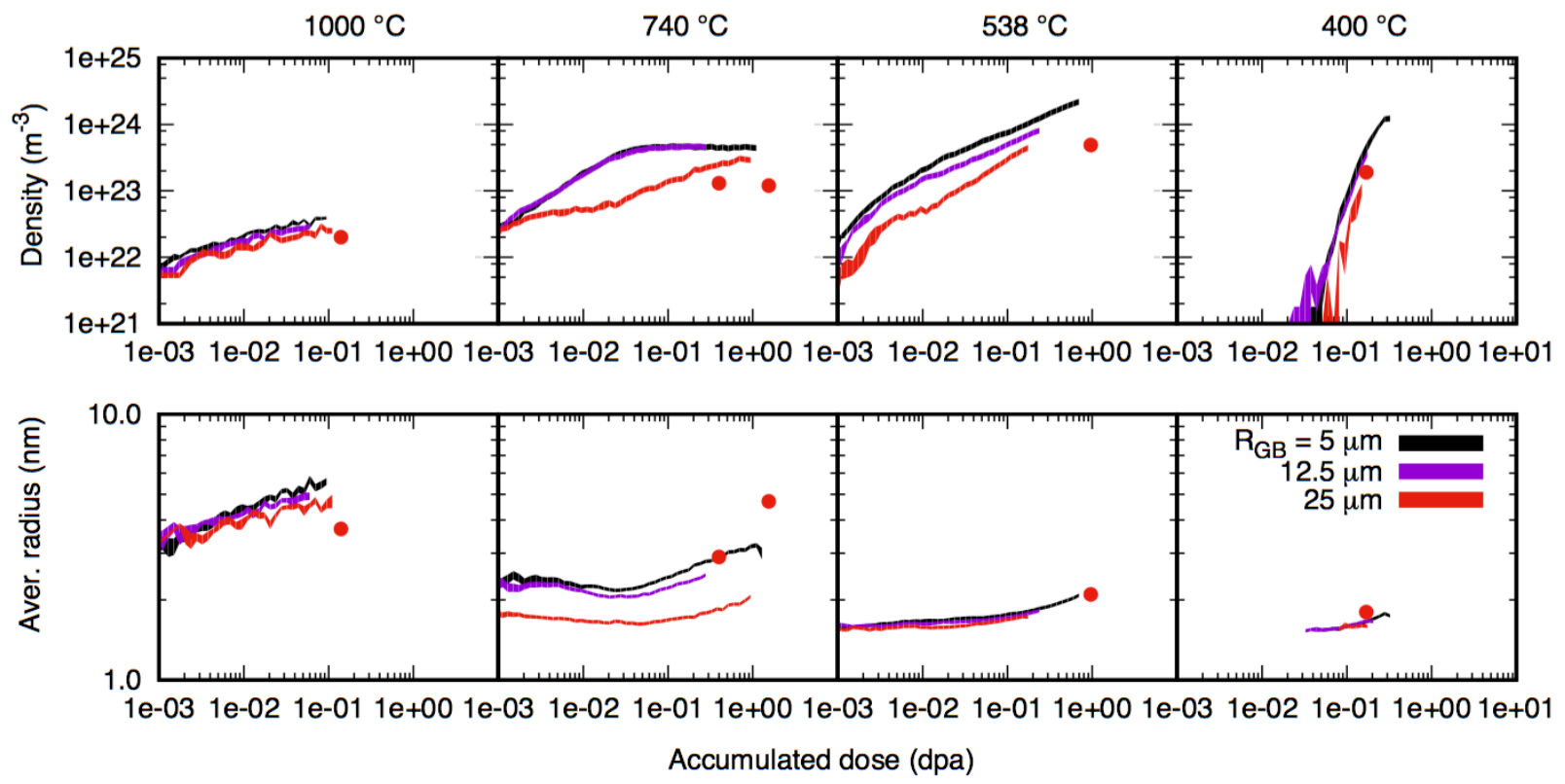

Figure 5: Evolution with the cumulated dose of the voids density and average void diameter found in our OKMC simulation box, as compared to experimental evidence $[14,15,22]$. Three different grain sizes $\left(R_{G B}\right)$ were assumed. In each figure, the line thickness corresponds to the standard error estimated from four independent runs.
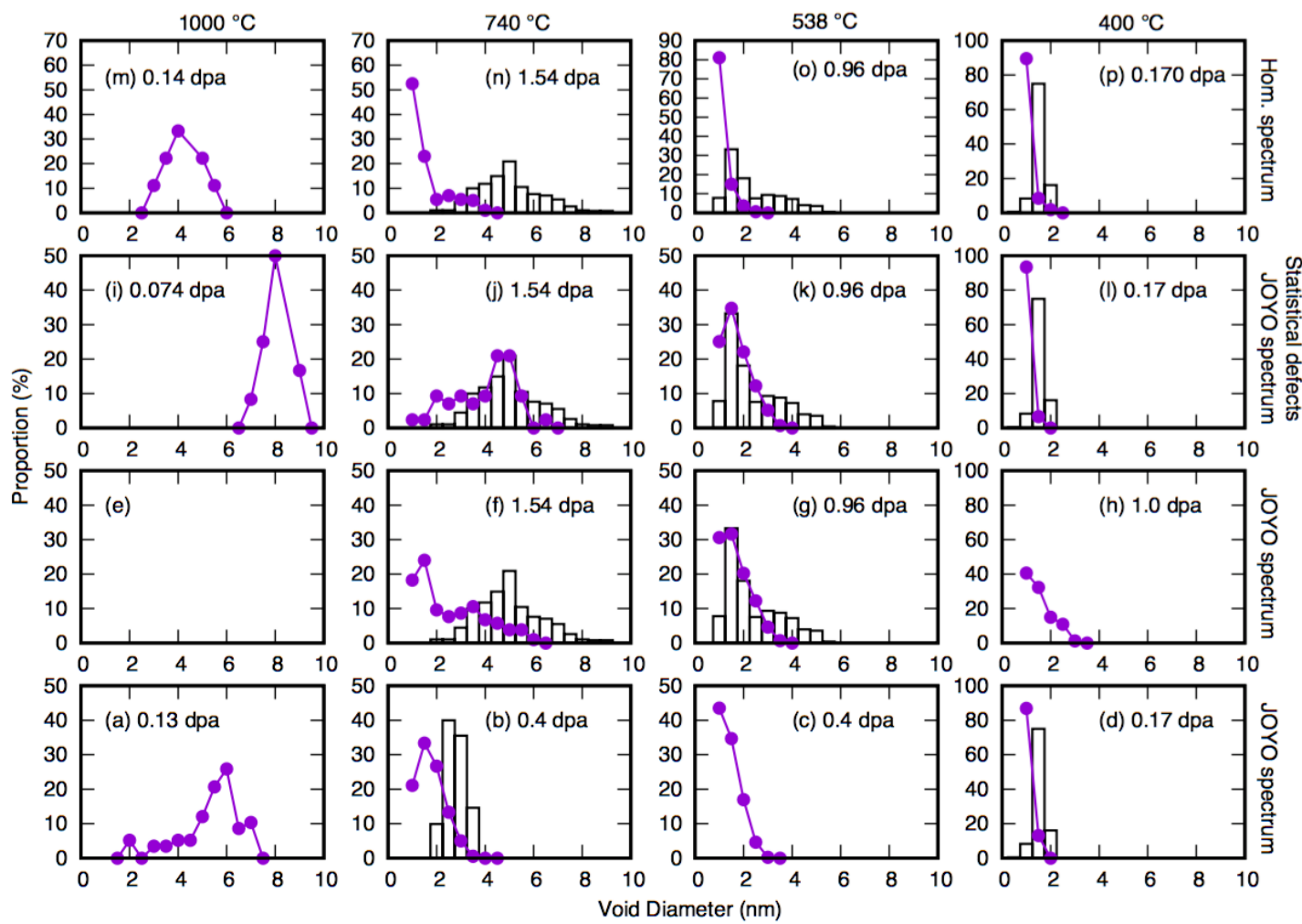

Figure 6: Comparison between the distribution of voids sizes as observed in TEM (histogram) and the prediction by our OKMC model (purple lines). All simulations where conducted assuming $R_{G B}=5 \mu \mathrm{m}$. Figures (a) to (n) on the bottom rows correspond to the reference case, assuming the JOYO spectrum for the PKAE and using the MD-based libraries of cascade debris for introducing defectsduring irradiation events. Figures (c) to (o) were obtained using the JOYO spectrum, but performing a statistical introduction of the cascade debris instead of using MD-obtained libraries. Figures (d) to (p) on the top row were obtained using the MD-based libraries, but assuming an homogeneous spectrum for the PKAE. 
is to find, given the dose, a lower density of voids and a higher average radius when the irradiation temperature is increased. This trend may be interpreted as a direct consequence of the increased mobility and decreased stability of vacancy clusters with increasing temperature. In other words, increasing the temperature dissolves the smallest voids easier (thus reducing their number because less of them reach the $1.5 \mathrm{~nm}$ size) and enhances the rate of coalescence (further reducing the density and directly increasing the average size). Note that the case of $400^{\circ} \mathrm{C}$ seems in contradiction with this trend, but it is due to the fact that the majority of the obtained voids are smaller than $1.5 \mathrm{~nm}$ before $0.2 \mathrm{dpa}$.

Looking back at Fig. 5, we see that the assumed radius for the grain boundary has non-negligible influence on the formation of voids, especially at $740^{\circ} \mathrm{C}$. Increasing the assumed grain size always reduces both the voids density and average radius. This translates the fact that the probability for SIA clusters to be absorbed at the GB is reduced, giving them more opportunities to recombine with vacancy clusters. Logically, we thus see a global reduction in the whole population of nanovoids, which appears in the figure via both their densities and average sizes. In spite of this variation, our results remain globally in satisfying agreement with the experimental evidence for the whole range of values explored, at the only exception of the $740^{\circ} \mathrm{C}$ case, where $R_{G B}=25 \mu \mathrm{m}$ seems to overly reduce the average radius.

Last, one should note that none of our simulations gave rise to the formation of big SIA clusters that could be assimilated to loops. This statement is in fact true for all the simulations presented through this paper, i.e., including in the next sections. Our understanding is that our OKMC model, as presently formulated, leaves indeed little chances for these big SIA clusters to form, because of the absence of elements in the tungsten matrix that are able to significantly slow down their fast diffusion towards either vacancy clusters or the grain boundary. Even the C-based trapping elements included in the next section are not strong enough to balance their intrinsic propensity to disappear from the simulation box between two irradiation events. A more complete model would include transmutation solutes (rhenium and eventually osmium) that are likely to attenuate SIA diffusion, as so would taking into account their long-range elastic interactions. Our results are, nevertheless, not contradictory to TEM observations, because the density of observed loops by Tanno et al. is in fact two orders of magnitudes lower than the void density; our simulation box is thus too small to expect even one loop to form.

\subsection{Influence of trapping elements}

In Fig. 7, our results as obtained including trapping carbonbased trapping elements in the 0-50 appm concentration range are shown, and compared with the original series in carbonfree tungsten. Clearly, and expectedly, these trapping elements have a strong influence on the obtained results. Not only do they largely delay the onset of nano-voids formation (by one or two orders of magnitude in dpa), but they also especially reduce their average size. Looking at the details of the sequence of events that were performed during the OKMC simulations, we understand this as a logical outcome from the reduction of thermal-activated diffusion of vacancy clusters; (a) During the second stage of the simulation as described in section 2.5, i.e., when no more fast-migrating SIA clusters are present in the simulation box and thus vacancy clusters have possibilities to perform migration events, a significant part of them get caught by trapping elements. Their diffusion is thus significantly lowered, meaning that the rate of coalescence is reduced, explaining why the average radius of the formed nanovoids decreases; (b) SIA clusters, when included in the simulation box after irradiation events, consequently find a higher density of smaller vacancy clusters counterpart in the matrix. The rate of recombination is thus enhanced, and the density of surviving vacancy clusters that manage to grow up to the nano-void size is thus reduced.

Globally, it is worth noting that the best agreement with experimental evidence is found in the absence of trapping elements, except for $1000^{\circ} \mathrm{C}$ where the inclusion of 20 appm carbon clearly shifted both the void density and the average void radius closer to the experimental point; the absence of diluted carbon atoms in the tungsten matrix gives rise to a higher density of oversized nano-voids, otherwise. At lower temperature, our description for the mobility and stability of vacancy clusters, complemented with no further assumption, spontaneously leads to relevant populations of nanovoids without the need of such trapping elements to moderate the rate of coalescence and thus enhance recombination. One can appreciate that the obtained results including 10-20 appm carbon do still compare well with TEM observations at $538^{\circ} \mathrm{C}$ and $740^{\circ} \mathrm{C}$, however, whereas the formation of nano-voids seems clearly delayed at $400^{\circ} \mathrm{C}$ even with only 10 appm carbon. Needless to say, our simplifying assumptions for the description of carbon, and the lack of accurate knowledge of its distribution in the real material, prevent us from objectively arbitrate on this question. The purpose of this work and the scope of the proposed model are clearly not to investigate the amount of dissolved carbon in the matrix, because the latter must instead be provided from either experimental evidence or from a precise model based on purely physical considerations. Determining an "optimal concentration" of our trapping elements to get the best comparison with the TEM data is thus inappropriate. Formally speaking, the conclusion we can draw from Fig. 7 is that our model is consistent with the complex and interlinked processes taking place in tungsten under neutron irradiation, and that our simplified assumptions about the particular role of carbon have apparently encompassed the limiting cases. While no quantitative estimation can be drawn, it is still worth noting that this "optimal carbon concentration", if estimated, would apparently increase with the irradiation temperature. One can appreciate that this is in fact sound from a physical point of view, consistently with the discussions in section 2.2, because it suggests that if most carbon atoms are supposedly found in grain boundaries or in other heterogeneous elements of microstructure at low temperature, the concentration dissolved in the matrix must logically increase with increasing temperature. 

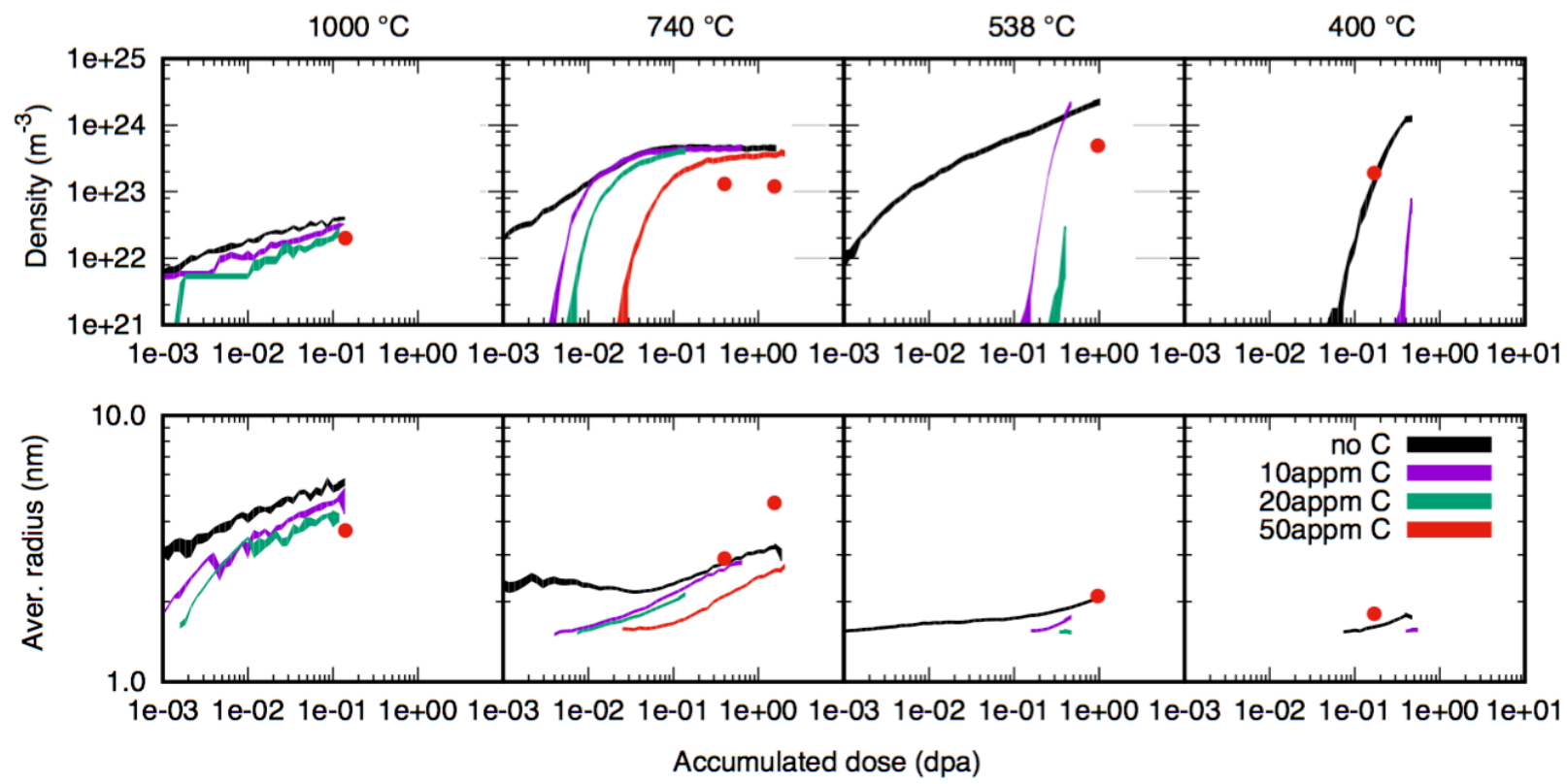

Figure 7: Evolution with the cumulated dose of the voids density and average void diameter found in our OKMC simulation box, as compared to experimental evidence $[14,15,22]$. Three different concentration of C-based trapping elements were included in the simulation box. In each figure, the line thickness indicate the standard error bar estimated from four independent runs.
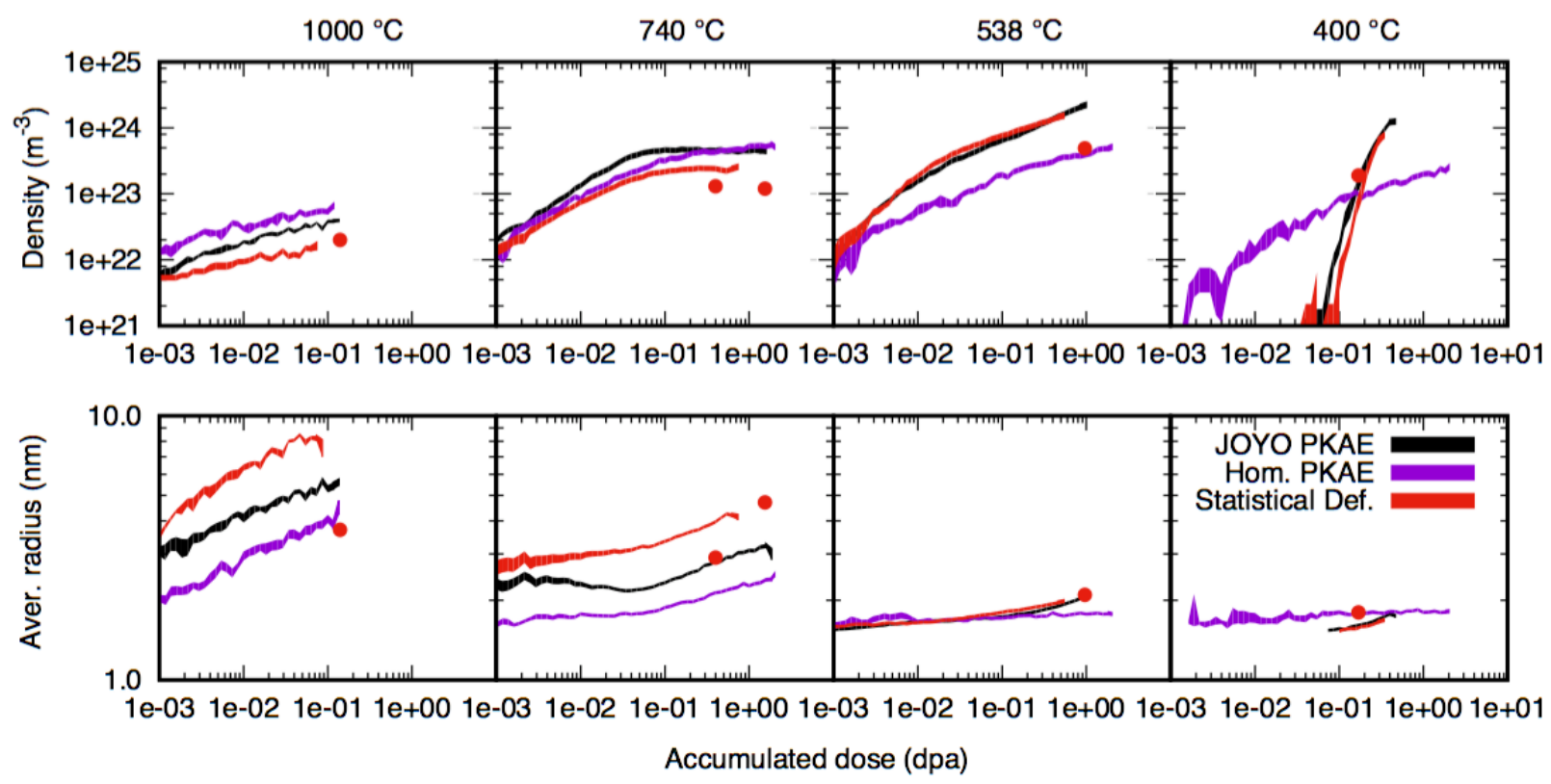

Figure 8: Evolution with the cumulated dose of the voids density and average void diameter found in our OKMC simulation box, as compared to experimental evidence $[14,15,22]$. Three series were obtained with different hypotheses in the description of neutron irradiation: "JOYO PKAE" uses the PKAE spectrum estimated for this reactor, whereas "homogeneous PKAE" assumes homogeneous spectrum, as shown in Fig. 2. Finally, "Statistical def." apply the statistical introduction of defects, as described in section 2.3. In each figure, the line thickness indicate the standard error bar estimated from four independent runs. 


\subsection{Sensitivity with respect to the description of irradiation}

In Fig. 8, our results as obtained using different models for neutron irradiation are shown. To start, we compare those obtained using the JOYO spectrum for the PKAE with those assuming an homogeneous spectrum. Generally speaking, in the second case, the formed nano-voids are always smaller, whereas the way their density is influenced seems to vary with the irradiation temperature. This latter point is in fact an artefact resulting from the choice for disregarding the voids smaller than $1.5 \mathrm{~nm}$, as can be appreciated comparing the cases (n) and (o) with cases (f) and (g) in Fig. 6. Visibly, assuming a homogeneous PKAE spectrum leads to the generation of many more voids, but their distribution in size is largely shifted towards the smallest ones (near the $1 \mathrm{~nm}$ ). The maximal size of voids reached is also significantly decreased. Looking back at Fig. 1 , we understand that it is a direct consequence of the highest PKAE in the spectrum, i.e., $E^{(P K A)} \geq 100 \mathrm{keV}$, for which clusters containing more than 33 vacancies (which corresponds to a diameter of $1 \mathrm{~nm}$ assuming a spherical shape) are found in the debris. The rate of seeding for stable nano-voids bigger than $1 \mathrm{~nm}$ is thus naturally enhanced, because they are directly included from collision cascades without the need for coalescence of various smaller vacancy clusters. Overall, these additional small voids prevent the growth of individual ones to larger sizes, because they compete in gathering the small clusters that coalesce with them after thermally activated diffusion.

Next, in Fig. 6 and in Fig. 8 we compare our results when using the MD-obtained libraries of collision cascade debris, or performing a "statistical introduction" of the same defects, i.e., discarding the information about their relative spatial coordinates and thus ignoring the effects of correlated recombinations imposed by the production bias, as discussed in section 2.3. One can see that in the second case, the obtained voids are characterized by a much lower density and a significantly larger size, except for the two lowest temperatures $\left(400^{\circ} \mathrm{C}\right.$ and $\left.538^{\circ} \mathrm{C}\right)$ where the same effect is negligible in magnitude. This translates the fact that in the absence of correlated recombination, many single SIA's are granted more opportunities for merging with other single SIA's or SIA clusters, with two consequences: by not being single SIA any more, not only do they loose their possibility for performing 3-D migration, but they also reduce their likeliness for getting recombined with vacancies (because the number of SIA's decreases). As a consequence, the surviving fraction of vacancies increases, and they tend to coalesce with existing voids, explaining why less and bigger ones form. These alterations, however, are damped at low enough temperature (lower than $740^{\circ} \mathrm{C}$ ), because, only the smallest vacancy clusters are mobile in these cases. Finally, it is worth mentioning that in addition to the results presented in Fig. 8, we also performed simulation in an intermediate case: instead of discarding all information concerning the relative positions and orientations of the SIA clusters, we also analysed our results when only the last information is ignored. In short, the results we obtained are almost indistinguishable from the "JOYO PKAE" series in Fig. 8, meaning that the most critical information to retain from MD simulations is the production bias.
To summarize, our results presented in this section highlight the importance of performing accurate modelling of neutron irradiation. Simplifying assumptions, such as ignoring the exact PKAE spectrum or the production bias in the collision cascades, may alter significantly the evolution of the microstructure with the irradiation dose.

\section{Summary and conclusive remarks}

In this work, we proposed an object kinetic Monte Carlo (OKMC) model for describing the evolution of the microstructure in polycrystalline tungsten under neutron irradiation. As a first step towards a future complete model, the focus was here given on technologically pure tungsten in the range of moderate doses (up to $1 \mathrm{dpa}$ ), thus ignoring the transmutation to rhenium and osmium that is pronounced above $\sim 0.5 \mathrm{dpa}$ (depending on thermal-to-fast neutron ratio). In its present state, our model accounts for the presence of carbon impurities, even though simplifying assumptions were taken in order to comply with the limited amount of input data available. Next, regular elements of the microstructure such as dislocations and grain boundaries are also included as stochastic events, consistently with the relatively small size of our simulation box. Neutron irradiation is also described as a stochastic event, for which the spectrum for the transfer of kinetic energy to the primary knock-on atom (PKAE), and the subsequent population of produced defects, is an explicit input parameter. The importance of an accurate description of the produced defects has been emphasized in the paper, which was compiled in this work thanks to the use of accurate libraries of cascade debris previously determined with molecular dynamics. In accordance with the available knowledge on the point defects and their clusters in tungsten, SIA clusters are mostly assumed to perform 1D migration, while vacancy-defects are naturally considered as $3 \mathrm{D}$ migrating objects. Mutual interaction of the defects is introduced on the basis of the capture radius, linked to the defect size. Dislocations and grain boundaries act as non-saturable sinks for both 1D and 3D migrating defects, whereas carbon atoms are trapping elements interacting with both SIA and vacancy defects.

The goal of this work was to perform a sensitivity study aiming to assess the quality of our model with respect to the accurate prediction of the void size/density distribution as a function of irradiation dose and temperature. The sensitivity study was therefore used to deduce the most critical elements in the model with respect to the onset of void swelling. We have shown that variation of grain size (in a physically justified range) has a limited impact on the onset of void swelling, and becomes significant only for the irradiation temperature $740^{\circ} \mathrm{C}$ and above. It's been noted that the variation of carbon content has a strong influence on the kinetics of the void formation, because carbonvacancy trapping significantly delays the growth of voids. Our results appear to have the best agreement with experimental TEM data, assuming that the concentration of carbon dissolved as interstitials inside grain's interior is in the range 0-20 appm. This does not exclude a presence of significant concentration of carbon located at grain boundaries, as typically commercially pure W may contain up to 200 appm (or higher). Because of the 
lack of direct experimental quantification, we have highlighted the importance to determine the concentration of carbon dissolved in the matrix (i.e., not in the segregated state), and we showed that our model is consistent with the natural assumption that the concentration of matrix-dissolved carbon should increase with temperature. At last, the sensitivity study demonstrated the importance of correlated recombination and accurate description of the PKAE spectrum, and thus intrinsically advocates for an accurate description of the primary irradiation defects using MD methods.

\section{Acknowledgements}

This work has been carried out within the framework of the EUROfusion Consortium and has received funding from the Euratom research and training programme 2014-2018 under grant agreement No 633053. The views and opinions expressed herein do not necessarily reflect those of the European Commission.

[1] S. Zinkle, Phys. Plasmas 12 (2005) 058101.

[2] S. Matsuda, K. Tobita, J. Nucl. Sci. Technol. 50 (2013) 321.

[3] M. Rieth, D. Armstrong, B. Dafferner, S. Heger, A. Hoffmann, M.-D. Hofmann, U. Jäntsch, Adv. Sci. Tech. 73 (2010) 11.

[4] S. Zinkle, J. Busby, Materials Today 12 (2009) 12.

[5] D. Stork, P. Agostini, J. Boutard, D. Buckthorpe, E. Diegele, S. Dudarev, C. English, G. Federici, M. Gilbert, S. Gonzalez, A. Ibarra, C. Linsmeier, A. L. Puma, G. Marbach, P. Morris, L. Packer, B. Raj, M. Rieth, M. Tran, D. Ward, S. Zinkle, J. Nucl. Mater. 455 (2014) 277.

[6] A. Giannattasio, Z. Yao, E. Tarleton, S. Roberts, Phil. Mag. 90 (2010) 3947.

[7] G. Pintsuk, Comprehensive Nuclear Materials 4 (2012) 551.

[8] A. Dubinko, A. Bakaeva, M. Hernández-Mayoral, D. Terentyev, G. D. Temmerman, J.-M. Noterdaeme, Phys. Scr. T167 (2016) 014030.

[9] D. Terentyev, A. Bakaeva, T. Pardoen, A. Favache, E. Zhurkin, J. Nucl. Mater. 476 (2016) 1.

[10] J. Coenen, S. Antusch, M. Aumann, W. Biel, J. Du, J. Engels, S. Heuer, A. Houben, T. Hoeschen, B. Jasper, F. Koch, J. Linke, A. Litnovsky, Y. Mao, R. Neu, G. Pintsuk, J. Riesch, M. Rasinski, J. Reiser, M. Rieth, A. Terra, B. Unterberg, T. Weber, T. Wegener, J. You, C. Linsmeier, Phys. Scripta T 167 (2016) 014002.

[11] H. Bolt, V. Barabash, W. Krauss, J. Linke, R. Neu, S. Suzuki, N. Yoshida, A. U. Team, J. Nucl. Mater. 329 (2004) 66.

[12] S. Zinkle, Fusion materials science: Overview of challenges and recent progress, in: 46th Annual Meeting of the Division of Plasma Physics of the American-Physical-Society.

[13] J. Steichen, J. Nucl. Mater. 60 (1976) 13.

[14] T. Tanno, A. Hasegawa, J. He, M. Fujiwara, S. Nogami, M. Satou, T. Shishido, K. Abe, Mater. Trans. 48 (2007) 2399.

[15] T. Tanno, M. Fukuda, S. Nogami, A. Hasegawa, Mater. Trans. 52 (2011) 1447.

[16] A. Hasegawa, M. Fukuda, S. Nogami, K. Yabuuchi, Fusion Eng. Des. 89 (2014) 1568.

[17] I. Gorynin, V. Ignatov, V. Rybin, S. Fabritsiev, V. Kazakov, V. Chakin, V. Tsykanov, V. Barabash, Y. Prokofyev, J. Nucl. Mater. 191 (1992) 421.

[18] M. Fukuda, K. Yabuuchi, S. Nogami, A. Hasegawa, T. Tanaka, J. Nucl. Mater. 455 (2014) 460.

[19] V. Jansson, M. Chiapetto, Malerba, J. Nucl. Mater. 442 (2013) 341.

[20] M. Chiapetto, L. Malerba, C. Becquart, J. Nucl. Mater. 462 (2015) 91, and references therin.

[21] C. Becquart, C. Domain, U. Sarkar, A. DeBacker, M. Hou, J. Nucl. Mater. 403 (2010) 75.

[22] R. C. Rau, R. L. Ladd, J. Moteff, J. Nucl. Mater. 33 (1969) 324.

[23] L. Malerba, C. Becquart, C. Domain, J. Nucl. Mater. 360 (2007) 159.

[24] V. Jansson, L. Malerba, A. DeBacker, C. Becquart, Domain, J. Nucl. Mater. 442 (2013) 218.
[25] X.-S. Kong, X. Wu, Y.-W. You, C. Liu, Q. Fang, J.-L. Chen, G.-N. Luo, Z. Wang, Acta Mater. 66 (2014) 172.

[26] T. Tanno, co authors, Private communication.

[27] H. ZHOU, S. JIN, Y. ZHANG, G. LU, Sci. China Phys. Mech. Astron. 54 (2011) 2164.

[28] W. M. Young, E. W. Elcock, Proc. Phys. Soc. London 89 (1966) 735.

[29] A. B. Bortz, M. H. Kalos, J. L. Lebowitz, J. Comput. Phys. 17 (1975) 10.

[30] K. A. Fichthorn, W. H. Weinberg, J. Chem. Phys. 95 (1991) 1090.

[31] V. Jansson, L. Malerba, J. Nucl. Mater. 443 (2013) 274.

[32] A. E. Sand, S. L. Dudarev, K. Nordlund, Europhys. lett. 103 (2013) 46003.

[33] A. Sand, K. Nordlund, S. Dudarev, J. Nucl. Mater. 455 (2014) 207.

[34] A. Stankovskiy, SCK-CEN, Belgium, kind contribution to this work.

[35] M. Gilbert, CCFE, United kingdom, kind contribution to this work.

[36] N. Castin, L. Malerba, J. Chem. Phys. 132 (2010) 074507.

[37] N. Castin, M. Pascuet, L. Malerba, J. Nucl. Mater. 429 (2012) 315.

[38] G. Henkelman, H. Jónsson, J. Chem. Phys. 113 (2000) 9901.

[39] M.-C. Marinica, L. Ventelon, M. R. Gilbert, L. Proville, S. L. Dudarev, J. Marian, G. Bencteux, F. Willaime, J. Phys.: Condens. Matter 25 (2013) 395502 . 